\title{
DETERMINATION D'UNE REPRESENTATION DES NOYAUX DE \\ VOLTERRA POUR UN SYSTEME PHYSIOLOGIQUE NON-LINEAIRE
}

M. Gautier, M. Monsion, J.P. Sagaspe

Université de Bordeaux I

Laboratoire d'Automatique

33405 Talence

\section{Introduction}

Nous cherchons un modèle de représentation du système crâniorachidien de 1 'homme à partir des enregistrements des variations de la pression intra-crânienne consécutives à des injections de liquide céphalo-rachidien artificiel dans les ventricules.

\section{Processus physiologique}

Le système crânio-rachidien est constitué par les 3 composants : tissus nerveux, système vasculaire, liquide céphalo-rachidien, contenus dans une enceinte rigide. La pression du liquide céphalo-rachidien ou pression intra-crânienne (P.I.C.) traduit l'état d'équilibre des trois composants et l'étude dynamique de son évolution apporte des données physio-pathologiques irremplaçables dans la compréhension des situations d'hypertension intra-crânienne chez l'homme.

De façon habituelle, trois tests sont pratiqués pour induire des variations de la P.I.C. :

- Compression bilatérale des veines jugulaires.

- Respiration d'un mélange de $\mathrm{CO}_{2}$.

- Injection incrémentale de L.C.R. artificiel à l'intérieur des ventricules.

Ces tests sont longs, délicats et parfois dangereux. Aussi avonsnous cherché à identifier globalement le système afin d'obtenir les mêmes rêsultats à partir de tests simples.

Le système peut être schématisé de la façon suivante :

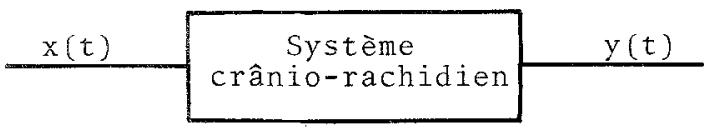


$x(t), 1$ 'entrée : injection de L.C.R. artificiel

$y(t)$, la sortie : P.I.C.

L'étude des enregistrements nous a montré que le système, déterministe, invariant, stable et continu, est non linéaire.

\section{Représentation de Volterra du système}

Compte tenu des propriétés du processus, il est possible d'approximer la relation fonctionnelle entrée-sortie, $y(t)=k[x(t)]$, par le développement en série de Volterra.

$$
\begin{gathered}
y(t)=\sum_{i=1}^{N} C_{i}\left[h_{i}, x(t)\right] \\
\operatorname{avec} C_{i}\left[h_{i}, x(t)\right]=C_{i}(t)=\int_{0}^{t} \ldots \int_{i}^{t} h_{i}\left(\tau_{1}, \ldots, \tau_{i}\right) \prod_{j=1}^{i} x\left(t-\tau_{j}\right) d \tau_{j}
\end{gathered}
$$

Ce développement peut être schématisé de la façon suivante :

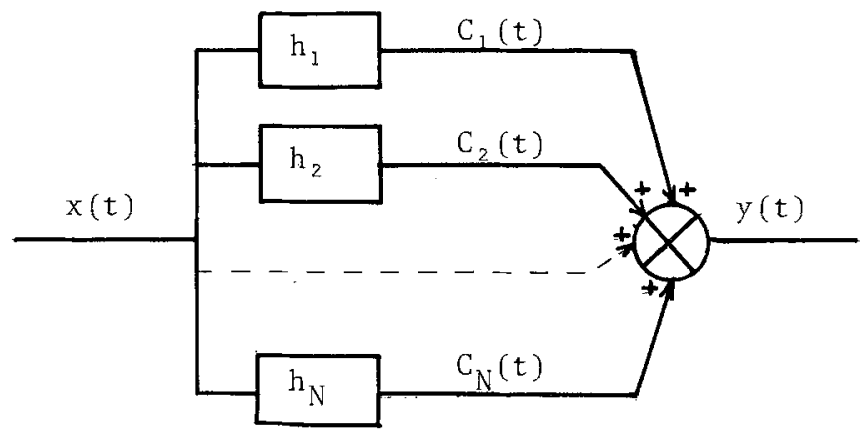

IV Détermination des fonctionnelles $\mathrm{C}_{i}$

En utilisant 1 a propriêté d'homogénéité des $C_{i}, C_{i}[\lambda x(t)]=$ $\lambda^{i} C_{i}(x(t)), \lambda \in \mathbb{R}$, il est possible de calculer les $N$ fonctionnelles $C_{i}(t)$ à partir de $N$ enregistrements des réponses $y_{1}, y_{2}, \ldots, y_{N}$ du système à des entrées $\lambda_{p} x(t)$

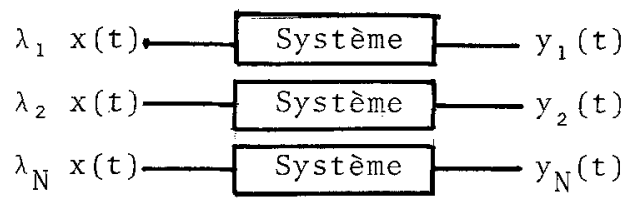


On utilise la méthode de Gardiner qui conduit à la relation :

$$
\left|\begin{array}{c}
C_{1}(t) \\
C_{2}(t) \\
\dot{C_{N}}(t)
\end{array}\right|=\left|\begin{array}{cccc}
\lambda_{1} & \lambda_{1}^{2} & \ldots & \lambda_{1}^{N} \\
\lambda_{2} & \lambda_{2}^{2} & \lambda_{2}^{N} \\
\dot{\lambda}_{N} & & & \vdots \\
\dot{\lambda}_{N} & \ldots & \dot{\lambda}_{N}^{N}
\end{array}\right|^{-1}\left|\begin{array}{c}
y_{1}(t) \\
y_{2}(t) \\
\vdots \\
y_{N}(t)
\end{array}\right|
$$

Notre problème se ramène donc à caractêriser $h_{i}$, noyau d'ordre $i$, connaissant l'entrée $x(t)$ et la sortie $C_{i}(t)$.

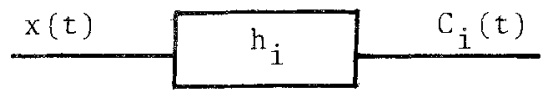

$V$ Caractérisation d'un noyau h

Sans nuire à la généralité de la méthode, nous développerons les calculs pour un noyau d'ordre deux et pour des entrées échelons.

Nous construisons un modèle $\hat{h}_{2}\left(\tau_{2}, \tau_{2}\right)$ de $h_{2}\left(\tau_{1}, \tau_{2}\right)$ sous la forme:

$$
\hat{h}_{2}\left(\tau_{1}, \tau_{2}\right)=\sum_{m=0}^{M} \sum_{n=0}^{M} B_{m n} g_{m}\left(\tau_{1}\right) g_{n}\left(\tau_{2}\right)
$$

Les fonctions $\left\{g_{i}(\tau)\right\}$ constituent une base orthogonale. $h_{i}\left(\tau_{1}, \ldots, \tau_{i}\right)$ est symétrique relativement aux permutations des $\tau_{i}$. Ceci implique que $B_{m n}=B_{n m}, \forall m$ et $n \in[0, M]$, et limite donc le nombre d'inconnues.

Nous obtenons ainsi une approximation de la sortie du noyau $h_{2}$ :

$$
\hat{C}_{2}(t)=\sum_{m=0}^{M} \sum_{n=0}^{M} B_{m n} \int_{0}^{t} g_{m}(\tau) d \tau \int_{0}^{t} g_{n}(\tau) d \tau
$$

La dernière étape consiste à déterminer les coefficients $B_{m n}$. Pour cela, nous décomposons $C_{2}(t)$ et $\hat{C}_{2}(t)$ sur une même base de fonctions orthonormales sur $[0, T]$ par rapport à la fonction poids $w(t)$.

$$
\begin{aligned}
& C_{2}(t)=\sum_{k=0}^{K} \gamma_{k} f_{k}(t) \text { avec } \gamma_{k}=\int_{0}^{T} C_{2}(t) f_{k}(t) w(t) d t . \\
& \hat{C}_{2}(t)=\sum_{k=0}^{K} \alpha_{k} f_{k}(t) \text { avec } \alpha_{k}=\int_{0}^{T} \hat{C}_{2}(t) f_{k}(t) w(t) d t .
\end{aligned}
$$

L'unicité de la décomposition nous permet d'assurer l'identité 
entre $C_{2}(t)$ et $\hat{C}_{2}(t)$ en écrivant $\alpha_{k} \equiv \gamma_{k}, \forall k=0, K$. Le calcul littéral de $\alpha_{k}$ nous fournit une relation $\alpha_{k}=R_{k}\left(B_{m n}\right)$. Nous obtenons ainsi un système linéaire dans lequel $B_{m n}$ sont les inconnues et que nous pouvons résoudre, compte tenu d'un choix convenable de $K$, ordre de la décomposition.

\section{Application}

Les fonctions de base sont des fonctions de Laguerre. Nous rappelons leurs propriêtés :

$$
\begin{aligned}
& \text { Dêfinition : } \Lambda_{n}(t)=(-1)^{n} \sqrt{2} e^{-t} L_{n}(2 t) \\
& \text { a vec } \\
& L_{n}(t)=\sum_{j=0}^{n}(-1)^{j} c_{n}^{j} \frac{t^{j}}{j !} \\
& \text { Orthonormalité sur }(0, \infty): \int_{0}^{\infty} \Lambda_{\mathrm{m}}(\mathrm{t}) \cdot \Lambda_{\mathrm{n}}(\mathrm{t}) \mathrm{dt}=\delta_{\mathrm{mn}} \\
& \text { Récurrence : }(n+1) \Lambda_{n+1}+(2 n+1-2 t) \Lambda_{n}+n \Lambda_{n-1}=0 \text {. } \\
& \text { Intêgrale: } I_{n}=\int_{\hat{q}}^{t} \Lambda_{n}(t) d t=\sqrt{2}-\Lambda_{n}(t)-2 \sum_{j=0}^{n-1} \Lambda_{j}(t)
\end{aligned}
$$

Décomposition de $\mathrm{C}_{2}(\mathrm{t})$

$$
C_{2}(t)=\sum_{k=0}^{K} \gamma_{k} \Lambda_{k}(t) \text { avec } \gamma_{k}=\int_{0}^{\infty} C_{2}(t) \Lambda_{k}(t) d t .
$$

Décomposition de $\hat{C}_{2}(t)$
Expression de $\hat{C}_{2}(t):$

$$
\begin{aligned}
& \hat{C}_{2}(t)=\sum_{m=0}^{M} \sum_{n=0}^{M} B_{m n} \int_{\theta}^{t} \Lambda_{m}(\tau) d \tau \int_{0}^{t} \Lambda_{n}(\tau) d \tau . \\
& \hat{C}_{2}(t)=\sum_{m=0}^{M} \sum_{n=0}^{M} B_{m n} I_{m}(t) I_{n}(t) .
\end{aligned}
$$

d'où la relation :

$$
\begin{aligned}
& \hat{C}_{2}(t)=\sum_{k=0}^{K} \alpha_{k} \Lambda_{k}(t) \text { avec } \alpha_{k}=\int_{0}^{\infty} \sum \Sigma B_{m n} I_{m}(t) I_{n}(t) \Lambda_{k}(t) d t . \\
& \alpha_{k}=\sum_{m=0}^{M} \sum_{n=0}^{M} B_{m n} \beta_{m n}^{k} \text { avec } \beta_{m n}^{k}=\int_{0}^{\infty} I_{m}(t) I_{n}(t) \Lambda_{k}(t) d t .
\end{aligned}
$$


Pour $k$ donné, nous obtenons une équation linéaire en $B_{m n}$

$$
\sum_{m=0}^{M} \sum_{n=0}^{M} B_{m n} \beta_{m n}^{k}=\gamma_{k}
$$

Le système obtenu en faisant varier $k$ de 0 à $K$ est soluble si: le nombre d'équations $K+1$ est supérieur ou égal au nombre d'inconnues $(M+1)(M+2) / 2$. Le tableau ci-dessous montre que 1 a dimension du système croît rapidement avec l'ordre i du noyau et $M$.

\begin{tabular}{|c|cccc|}
\hline M & 1 & 2 & 3 & 4 \\
\hline 1 & 2 & 3 & 4 & 5 \\
2 & 3 & 6 & 10 & 15 \\
3 & 4 & 10 & 20 & 35 \\
\hline
\end{tabular}

Ceci constitue le seul inconvénient lors de l'implantation sur ordinateur. En effet, les coefficients $\beta_{\mathrm{mn}}^{\mathrm{k}}$ peuvent être calculés à priori puisqu'ils ne dêpendent que du choix de la base et du type d'entrée. Le calcul des $C_{j}(t)$ et des $\gamma_{k}$ se réalise facilement et 1 'on peut dire que la méthode est globalement très rapide.

\section{Conclusion}

Nous proposons une méthode de détermination des noyaux de Volterra d'un système non linéaire déterministe, invariant, stable et continu. La rapidité des calculs et la simplicité des tests nécessaires en font un outil pratique et efficace dans l'aide au diagnostic relatif aux cas d'hypertension intra-crânienne. 


\section{BIBLIOGRAPHIE}

1 - GaUtier m., MONSiOn M., SAgaspe J.P.

Caractérisation des noyaux de Volterra d'un système non linéaire. Electronic Letters. Vol $11 \mathrm{n}^{\circ} 15 \mathrm{p} .351$ à 353.

2 - GARDINER A.

Identification of processus containing single-valued non-linearities. Int. J. Control, 1973, 18, n ${ }^{\circ} 51029$ à 1039 .

3 - VOLTERRA $V$.

Theory of functionals and of integral and integro-differential equations.

New-York, Dover. 